\title{
Text Input Error Categorisation: Solving Character Level Insertion Ambiguities Using Zero Time Analysis
}

\author{
Akiyo Kano \\ Department of Computing \\ University of Central Lancashire, Preston, \\ PR1 2HE, England \\ +44-1772-89-5300 \\ akano@uclan.ac.uk
}

\author{
Janet C Read \\ Department of Computing \\ University of Central Lancashire, Preston, \\ PR1 2HE, England \\ +44-1772-89-3285 \\ Jcread@uclan.ac.uk
}

\begin{abstract}
A review of literature on text input error categorisation revealed the need for a formal method to assist in solving ambiguities. This paper proposes a method of solving one such set of ambiguities, those caused by insertion of an extra letter. The method uses two rules: the Zero Time rule and Impossible NT/CT-Mu rule to establish whether the extra letter was inserted with another letter, or inserted individually. The method was applied to two large studies conducted to gather typing errors from students and children. The results show that the method is able to solve $100 \%$ of all insertiononly ambiguities and in doing so it helps reduce ambiguities in $75-85 \%$ of the remaining ambiguities.
\end{abstract}

\section{Categories and Subject Descriptors}

H.5.5 [Information Interfaces and Presentation (e.g., HCI)]: User Interfaces - input devices

\section{General Terms}

Measurement, Experimentation, Human Factors.

\section{Keywords}

Typing Errors, Insertion, Ambiguities, Solving Ambiguities, Timestamp, Key Log.

\section{INTRODUCTION}

Text input refers to the process by which text is inputted to a computer. MacKenzie and Tanaka-Ishii [16] list several text input methods. One method is a keyboard based text input, which uses key presses to input text. The keyboard can be full sized (like a QWERTY keyboard) or can be reduced (as found on mobile phones). Full-sized keyboard text entry is generally referred to as typing, a process that has been well studied $[3,4,16]$.

Text entry research has a history dating back to before computers, to when the keyboard was created for typewriters in the early $20^{\text {th }}$ Century [2]. Researchers have since studied text input methods in order to evaluate new products and test for further improvements in efficiency. Text entry research has evolved with the advance of technologies that allowed the researchers to investigate how users input text in more detail.

(C) The Author 2009.

Published by the British Computer Society
At its earlier stages, text input analysis consisted of a researcher with a stopwatch, and a participant at the typewriter. In contrast, researchers now have key logging software and timestamps to record what participants type, to capture any mistakes that were corrected during the typing, and to $\log$ the times that each key was pressed. These additional data allow researchers to solve ambiguities and decipher how the error was created with much greater accuracy.

\subsection{Typing Error Data Collection}

Typing error analysis commonly involves data collection by phrase copying tasks. The participants are shown short, easy to memorise phrases and asked to type the phrases using the typing method(s) under investigation. In most cases, software logs the keys pressed and its timestamp. Phrase copying is preferred as it allows researchers to know what the participant intended to type $[11,15,23]$.

There are two approaches to analysing the data collected in such tasks. The first is based on error rates and participant efficiency. In this model, counts are made of the number of errors committed, the number of errors corrected, and the number of errors left uncorrected. The effort expended by the participant is calculated by keystrokes, or keystrokes per character and the time taken. Error rates are easy to compute, but offer little information as to how the error occurred. The second approach is to consider the different errors made and attempt to classify them in some way. This latter approach is used when the cause of the error needs to be understood, as it offers details of what sort of error has occurred.

Central to both approaches is the comparison of two text strings, the presented text (that which was being copied or was intended to be created) (PT) and the transcribed text (that which appears) (TT). To calculate the error rate, these two strings are lined up using an MSD (Minimum String Distance) algorithm [13, 21]. For example, consider the following PT and TT:

$$
\begin{aligned}
& \text { PT: the quick brown fox } \\
& \text { TT: tha quck browz fox }
\end{aligned}
$$

The MSD for the above example is 3 , since TT requires a substitution ('a' to 'e'), an insertion (of ' $\mathrm{i}$ ') and an omission (of ' $z$ ') to return back into PT.

Once the strings are aligned, the errors can be counted, and later investigated. A base error rate is derived by counting the minimum number of primitives (insertions, deletions or substitutions) needed to transform one string into the other. This error rate thus shows how far the transcribed text is from the intended text. To categorise the errors, the PT is compared with either the TT or the Input Stream (IS) - all the keystrokes produced during the task - and following 
definitions of several error types, each error is matched to an error type. Whereas calculating error rate is a simple calculation, categorising errors into predefined types is dependent on the categorisation method used, more difficult and prone to ambiguities.

\section{CATEGORISATION METHODS}

Categorisation methods list definitions of error types that can be made by the participants. There are many categorisation methods, often tailored to count only the error types that the researcher is interested in. These methods have originated in the field of Psychology $[5,14]$ since the early $20^{\text {th }}$ century, and are often used to understand the psychological process of typing. They consider what the errors could indicate about their causes, and construct mental models of typing. These methods usually group error types by the estimated source of the errors, for example, temporal, spatial and execution errors. The field of HCI, which followed later on, uses such methods to compare one text input device to another. Therefore, the errors are often defined and grouped in relation to the hardware, such as the NT/CT errors [12] defined later. Table 1 summarises some of the categorisation methods found in the literature from 1945 to 2007.

In instructing typists to increase accuracy with specific typing drills, White [22] highlights twelve error types. As White's text was designed to help typists, it did not formally define these errors, but an analysis of the training drills can offer some clues as to the definitions of these errors.
Dvorak et al. [4] investigated White's work and defined several other error types. These included Adjacent Errors, which are errors where the keystroke is falsely directed to an adjacent key, such as typing 'amvition' for 'ambition'. A second error type is Copy-Reading Errors, which occur when the word was (assumed to be) read incorrectly from the presented text. An example given by Dvorak et al. is the typing of 'admiration' for 'ambition'. Two transposition error types are described, the first is the basic Transposition Error, defined as an interchange of two strokes; a Transposed Doubling error is one where two such errors occur, for instance in the typing of 'thses' for 'these'. Although lacking formal definitions in the book, other classifications, including Omitting Word, Substitution, and Omission, are also mentioned.

In understanding the serial order model of typing, MacNeilage [17] tested 5 female college students who were touch typists, all at average professional speed (30-45 words per minute). Subjects typed up rough reports at home, which were analysed for 623 errors. He grouped his error types into Spatial (where the error was related to where the keys were on the keyboard) and Temporal (errors in the order in which the required letters were typed). He concluded that Spatial Errors mostly associated with the left hand and keyboard difficulty, while Temporal Errors mostly arose from language structure variables.

In Spatial Errors, MacNeilage defined Horizontal Errors (typing a letter immediately to the left or right of the correct letter), Vertical Errors (typing a letter immediate above of

Table 1: Summary of Typing Error Categorisation Methods from 1945 to 2007

\begin{tabular}{|c|c|c|c|c|c|c|c|}
\hline & White [22] & Dvorak et al. [4] & $\begin{array}{l}\text { MacNeilage } \\
{[17]}\end{array}$ & $\begin{array}{l}\text { Gentner et al. } \\
{[6]}\end{array}$ & Logan [14] & $\begin{array}{l}\text { Wobbrock \& } \\
\text { Myers [23] }\end{array}$ & Kano [12] \\
\hline Insertion & $\begin{array}{l}\text { Inserted Strokes, } \\
\text { Double strokes, } \\
\text { Syllable Division, } \\
\text { Repeating words }\end{array}$ & & $\begin{array}{l}\text { Interpolation } \\
\text { Error }\end{array}$ & $\begin{array}{l}\text { Insertion, } \\
\text { Misstrokes }\end{array}$ & $\begin{array}{l}\text { Immediate, } \\
\text { preservation, } \\
\text { Space bar, } \\
\text { separation, } \\
\text { Character } \\
\text { separation, } \\
\text { Letter Sequence, } \\
\text { Error Habits, } \\
\text { Home-letter } \\
\text { intrusion }\end{array}$ & Insertion & $\begin{array}{l}\text { CT-Mu, DL, } \\
\text { DS, ExE, IF, } \\
\text { IL, IS, ISy, } \\
\text { NT-Mu, IW- } \\
\text { A, } \\
\text { IW-U, DW, } \\
\text { DP, EE }\end{array}$ \\
\hline Omission & $\begin{array}{l}\text { Letter Omission, } \\
\text { Omitting Words }\end{array}$ & $\begin{array}{l}\text { Omission, } \\
\text { Omitting words }\end{array}$ & Omission errors & Omission & $\begin{array}{l}\text { Letter, } \\
\text { Syllable, } \\
\text { Word, } \\
\text { Space bar, }\end{array}$ & Omission & $\begin{array}{l}\text { OL } \\
\text { OS } \\
\text { OW } \\
\text { OP } \\
\end{array}$ \\
\hline Substitution & $\begin{array}{l}\text { Substitution, } \\
\text { Transposition, } \\
\text { Capitalisations, } \\
\text { Word substitution, } \\
\text { Adjacent letter } \\
\text { substitution }\end{array}$ & $\begin{array}{l}\text { Substitution, } \\
\text { Adjacent Errors, } \\
\text { Copy Reading, } \\
\text { Transposition, } \\
\text { Transposed } \\
\text { Doubling }\end{array}$ & $\begin{array}{l}\text { Horizontal, } \\
\text { Vertical, } \\
\text { Diagonal, } \\
\text { Reversal, } \\
\text { Anticipation, } \\
\text { Phonemic, } \\
\text { Type errors, } \\
\text { Contralateral }\end{array}$ & $\begin{array}{l}\text { Substitution, } \\
\text { Transposition, } \\
\text { Interchange, } \\
\text { Migration, } \\
\text { Doubling, } \\
\text { Alternating }\end{array}$ & $\begin{array}{l}\text { Remote, } \\
\text { Horizontal, } \\
\text { Vertical, } \\
\text { Number sub, } \\
\text { Homologous, } \\
\text { Hand position, } \\
\text { Transposition (1 } \\
\text { finger, } 2 \text { fingers, } \\
2 \text { hands), } \\
\text { Interchange, } \\
\text { Migration, } \\
\text { Alternation, } \\
\text { Doubling, } \\
\text { Another word, } \\
\text { Perseveration }\end{array}$ & Substitution & $\begin{array}{l}\text { AE } \\
\text { CE } \\
\text { CT-S } \\
\text { DE } \\
\text { IE } \\
\text { NT-S } \\
\text { SL } \\
\text { TE } \\
\text { ME } \\
\text { SW-A } \\
\text { SW-U } \\
\text { SP }\end{array}$ \\
\hline Other & Spacing & & $\begin{array}{l}\text { Equivocal, } \\
\text { Multiple } \\
\text { classification, } \\
\text { Unclassifiable }\end{array}$ & & Spelling & & $\begin{array}{l}\mathrm{U} \\
\text { ExE } \\
\mathrm{CNE} \text { (error) }\end{array}$ \\
\hline
\end{tabular}


below the correct letter in the same column of the keyboard), and Diagonal Errors (a letter is typed in a row and a column adjacent to that of the correct letter). He noted that for these errors, it cannot be shown whether the error was made due to the user hitting the wrong key, or using the wrong finger.

In Temporal Errors, he defined Reversal Errors (two letters next to each other in the correct sequence are reversed in their order), Omission Errors (one letter in a sequence is left out), Equivocal Errors (the letter one stroke ahead of the one required in the copy is typed, after which the subject stops), and Anticipation Errors (a letter is typed which is required more than one stroke ahead of the place where it is mistakenly typed). He also defines a group of miscellaneous errors such Interpolation, Phonemic Errors, Type Errors, Contralateral Errors, Dynamic Errors, Multiple Classification Errors, and Unclassifiable Errors.

A major work in studying typing was carried out by Cooper et al. [3], in which Gentner et al. [7], formally defined and listed nine different error types as summarised in Table 1. Note that the term Transposed Doubling Error defined by Dvorak et al. [4] was described by Gentner et al. as an Alternating Error, which perhaps better describes the nature of the error.

Logan [14] carried out a longitudinal study on one professional typist across two decades. He believed that analysing a large number of errors created by one person would increase our understanding of the processes and mechanisms underlying skilled copy typing. He collected 3000 errors (out of approximately 1,300,000 keystrokes) and classified them into 27 error types. Logan based his categorisation on Gentner et al. [6] and MacNeilage's work, [17] and his contribution is regarded as an expansion of these methods.

Wobbrock and Myers [23] took error classification back to its basic level by classifying errors into Insertion, Omission and Substitutions only, and whether they were corrected or uncorrected. Wobbrock and Myers defined an Insertion as occurring when a letter appears in TT, but not in PT. Omission occurs when a letter appears in PT, but not in TT. Substitution occurs when the corresponding letter in PT and TT does not agree. Examples of these definitions are shown below (PT shown in brackets):

Insertion: coerrection (correction)

Omission: corection (correction)

Substitution: corrwction (correction)

At this basic level, the method loses the detail of what errors were made as more complex errors are categorised as several primitive errors [12]. For example, a Migration error, such as shown below where the first ' $c$ ' migrates across 3 letters:

orrcection (correction)

is classified as Omission of the first 'c', then an Insertion of the ' $c$ ' later in the word. This loss of detail hinders the effort of the researcher in understanding how the error occurred, and so it is common practice to include further error types. However, several other researchers also use Insertion, Substitution and Omission only, due to its simplicity [1].

Kano et al. [12] gathered 1060 typing errors from 112 children. From these errors, they merged error types found in the literature with several new error types not previously defined. Most significantly, they extended on White [22] by introducing more word level errors and even phrase level errors. These error types are concerned with errors on a larger scale. The Kano et al. classification method has 33 error types, grouped into letter, word and phrase level errors (see Table 2).

Table 2: Summary of Kano et al. [12] Method

\begin{tabular}{|l|l|l|l|l|}
\hline & Omission & Substitution & Insertion & Other \\
\hline \hline Letter & OL & AE & CT-Mu & U \\
& OS & CE & DL & ExE \\
& & CT-S & DS & CNE(error) \\
& & DE & ExE & \\
& & IE & IF & \\
& & NT-S & IL & \\
& & SL & IS & \\
& & TE & ISy & NT-Mu \\
\hline Word & OW & SW-A & IW-A & \\
& & SW-U & IW-U & \\
& & & DW & \\
\hline Phrase & OP & SP & DP & \\
& & & EE & \\
\hline
\end{tabular}

As the classification methods become more thorough, the increased number of error types allows for more errors to be detected. However, this also increases the number of errors that could be categorised into multiple types - i.e. it increases the potential for ambiguity. Due to the lack of formal rules in solving ambiguities, these errors are often arbitrary categorised, often varying between those people classifying the errors.

It should be noted that these classification methods are not exhaustive and do not capture errors in their entirety. Categorising to only a few error types reduces the resolution at which errors can be analysed, whereas large number of error types are prone to ambiguities. Salthouse [20] states in his categorisation of Substitution, Insertion, Omission and Transpositions that: "they are not to be considered exhaustive, but rather as a representing classifiable patterns of keystrokes that, when taken together, encompass a large proportion of misstrokes".

\section{INSERTION ERROR TYPES AND AMBIGUITIES}

Although ambiguities can occur in the classification of any error types, the scope of this paper is in resolving ambiguities within insertion errors. The following section outlines insertion errors defined in literature, detailing how insertion errors can be ambiguous and discuss how others have attempt to deal with such ambiguities.

\subsection{Insertion Error Types}

Insertion errors are one of the most frequent mistakes made by typists $-42 \%$ of errors found in Grudin [8] and 36\% of errors found in Salthouse $[18,19]$ were insertion errors. As such, many researchers have sought to more carefully define this error type. Some count any character or a symbol inserted as an insertion error while others subdivide into further error types according to what letter was inserted. Insertion error types found in literature can be grouped into those letters inserted on their own, and those that produce more than one letter with a single keystroke.

\subsubsection{Insertion Involving Single Letters}

One of the earliest definitions of an insertion error was by Book in 1925 [2]. He analysed errors made by contestants of the 1923 International Championship Typewriting Contest, 23 
participants of various skill levels, from professionals to novices. He defined 3 insertion errors: addition or insertion of letters, space-bar strokes, and in the expert stage of typing, insertion of extra words.

MacNeilage [17] studied 5 female university students, collecting 442 classifiable errors, defined into twelve error types. He grouped the error types into Spatial, Temporal and Miscellaneous errors. He has no insertion errors under Spatial, but defines Equivocal and Anticipation under Temporal and Interpolation under Miscellaneous. Equivocal Errors occur when the letter one stroke ahead of the one required in the copy is typed, after which the subject stops (becoming aware of the error); e.g. 'stiml—' for 'stimulus'. The error was named equivocal because it shows some conformity to Reversal Errors and Omission Errors but, since the subject stopped immediately after making it, cannot be categorised as either. Anticipation Errors occur when a letter is typed which is required more than one stroke ahead of the place where it is mistakenly typed; e.g. 'ext-' for 'expected'. Interpolation occurs when a letter apparently quite unrelated to the correct sequence is inserted in it; e.g. 'formend' for 'formed'.

Gentner et al. [6] defined two insertion error types. Insertion Errors are an extra letter inserted into a text. Some insertions can be classified as Misstrokes errors. Misstroke errors can be traced to inaccurate motion of the finger, such as when one finger strikes two keys simultaneously.

Salthouse [18] named insertion errors as Intrusion Errors and defined these as 'a character was added (e.g. wored for word)' but only if the inserted letter was the only erroneous keystroke.

Logan [14] grouped six insertion error types into Insertion and Intrusion Errors. Under Insertion (extra letter) errors group he lists: Preservation Errors, which are immediate insertion of a repeated letter in the text (e.g. 'difffer'), some of these involved repeating letters that are never double in the text (e.g. 'phhenomenon'). Space Bar Separation, where a word is separated by an insertion of a space, and Character(s) Separation where a word is separated by another character (e.g. 'tetndency'). Logan does not define in detail the errors grouped under Intrusion Error, but offers some examples: Letter Sequence ('undere' for 'under'), Error Habits ('undter' for 'under') and Home-Letter Intrusion where the touch typist inserts a letter from the home row ('sufflicient' for 'sufficient').

Wobbrock and Myers [23] define Insertion as extra characters in TT (transcribed Text) or IS (Input Stream) that lack a corresponding character in PT (Presented Text). They further categorise this into those where there has been an attempt to correct, but not necessarily fix (Corrected Insertion), and those that no attempt was made to correct (Uncorrected Insertion).

Kano et al. [12] offer six insertion errors that involve one letter, and three insertion errors that produce more than one character. This method is so far, although not complete, the most thorough classification of insertion errors. In errors that involve one letter, these were refined according to what letter was inserted. Inserted Letter (IL) refers to an extra letter (not a duplicate of the previous letter) that is inserted (e.g. 'hern' for 'her'). Duplicated Letter (DL) is when a character is erroneously repeated twice in a row, such as when 'always' is typed as 'alwaays'. Inserted Space (IS) is when an extra space is inserted where there should be no spaces according to the intended text (e.g. 't eam' for 'team'). Duplicated Spaces (DS) refers to an error when two spaces are typed where only one space is shown in the presented text (e.g. 'these cookies' for 'these cookies'). Inserted Symbol (ISy) errors are symbols that are inserted when there are no symbols in the presented text. Inserted Function (IF) refers to a function key, such as Control and Alt being pressed when not required.

\subsubsection{Insertions Producing Multiple Letters}

Insertion error types so far outlined do not take into account that in most modern text input devices it is possible to press more than one key at a time. This is partly due to the main body of work in the field being related to typewriters, where the typing mechanisms made it very difficult to produce two key presses at the same time. However, with no typebars jamming, the electronic keyboard allows this to happen frequently. During his video analysis of novice and expert typists, Grudin [8] comments that more than one key can be pressed, and reports that most intrusion errors examined involved two keys being struck by the same finger.

Kano et al. [12] defined these insertion errors that involve more than one letter. Next To Error Multiple Key Presses (NT-Mu) refers to when a key directly next to the intended key is pressed along with the intended key, producing the intended letter and one or more extra letters. Some examples of NT-Mu error are 'ourt' for 'our', and 'agwes' for 'ages'. Close To error Multiple key presses (CT-Mu) refers to when one or more keys 'close to' but not next to the intended key are pressed together with the intended key, producing the intended letter and one or more extra letters. Some examples are 'onl7y6' for 'only' and 'wr8iting' for 'writing'. It is possible for NT-Mu or CT-Mu errors to be another error type created from a spelling mistake (such as IL error), but when the extra letter is a NT or CT letter to the intended letter, it is classified as a NT-Mu or CT-Mu error.

From these definitions, it is clear that an insertion error can occur in two ways. The first is when the typist presses two keys at the same time. The second is when the person intended, incorrectly, to type an extra letter in between two intended letters.

\subsection{Ambiguities}

When classifying typing errors, it is common for some errors to be attributed to several causes and hence placed in more than one category $[12,14,17]$. This is particularly true in corrected errors where the errors were corrected immediately. This situation is exacerbated during manual classification, where the error and the error definitions are open to interpretation, causing different classifications between the categorisers. Logan [14] admits that he classified errors based on arbitrary decisions, and thus his results are only an approximation of the frequencies with which these types of errors occurred. For example, typing 'ero' for 'error' might be an omission rather than temporal error. With no way of knowing exactly which error type it was, frequency for both error types can only be an approximation.

\subsubsection{Resolving Ambiguities}

One common method used to reduce ambiguities errors that do not fit into the method definitions is to not count them. MacNeilage [17] ignored any errors that were simple spelling mistakes (how he decided that is unknown). Any error involving more than one letter was classified Unclassifiable, as these errors were more likely to fit into several error types. 
MacNeilage also had a unique error type for those errors remaining, which still could fit into more than one error type, called as Multiple Classification Error.

In an effort to reduce misclassifications and ambiguities, two studies used a second researcher to categorise the errors independently $[12,17]$. In both studies, the main investigator manually categorised the errors, then a second researcher classified the errors independently. Where there was any difference in the categorisation, they discussed suitable error types until both parties were satisfied. MacNeilage [17] found approximately $70 \%$ were classified into single error type, $10 \%$ were unclassifiable and $20 \%$ remained with a multiple classification.

These methods are both limiting in the number of errors they could classify, and in their accuracy to classify errors correctly. Rather than trying to solve ambiguities in an arbitrary manner, disambiguation methods must follow formal structured rules. Wobbrock and Myers [23] offer a classification method that attempts to solve ambiguities with algorithms. When a complex typing error involves several errors, their program offers all the possible combinations of errors. As the classification is carried out algorithmically, there are no biases to certain error types or misclassifications. However, when presented with several possible combinations, this method does not specify which combination is most likely. This method is also only suitable for any techniques that produce one character at a time, assuming that it is not possible to produce two key presses in the same keystroke. However, as stated already, it is possible for two keys to be struck by one keystroke. This research attempts to solve these ambiguities using timing at which a letter is inserted.

\subsubsection{Key Loggers and Timestamps}

To discover whether two keys were pressed at the same time, it is crucial to look at the times at which they were pressed. To do this, we need to use some form of logging the keys with timestamps.

Key loggers are often used in text input experiments, to record what keys were pressed. This allows the researcher to see all the keys that the participant pressed, including those keys that were subsequently deleted during error correction. However, not all key loggers log the time at which they were pressed.

In the early period of text input research, hardware key loggers were used for keystroke logging by means of a hardware circuit that was attached somewhere in between the computer keyboard and the computer. In modern times, software key loggers are most commonly used. They record the timestamp of each key pressed and allow researchers to see how long a participant took to complete a typing task and time taken between each keystroke. Timestamps of key logs has also been incorporated into original text input test software created by text input method researchers [23].

\subsubsection{Extracting Data From Timestamps}

Timestamps have been widely used in text input analysis for various purposes. Hayes et al. [9] used timers to measure reaction time of each finger to light or character signals, to study the relation between the reaction time and typing rates of 24 typists. He recorded these on mechanical interval timers to the nearest 0.001 second. In analysing the standard QWERTY keyboard, Hiraga et al. [10] measured timing between each keystroke to see if certain keying sequences were easier than others. They theorised that if the timing between each key press is shorter for a particular key sequence, the sequence must be easier to type. They too measured time to nearest 0.001 second. Grudin [8] used video and timestamps to calculate the timing of key presses. He analysed behavioural differences between experts and novices ( 8 novices and 6 experts) and found that most Intrusion Errors examined involved two keys struck by the same finger. This led him to conclude that the error was in the execution, because it is assumed to be the result of faulty implementation of the key press. Salthouse [20] found that 54\% of his Intrusion Errors were adjacent letters to the intended key. $\mathrm{He}$ used timing to investigate pauses in typing around Intrusion Errors and found that when an adjacent key is struck with the intended key, the timing between the two key presses were much shorter (less than 0.1 second) than average. He thus concluded that adjacent insertions occurred by a finger being incorrectly positioned between two keys, resulting in the two keys being pressed together.

\section{THE REAZON METHOD}

The new method (ReAZoN - Resolving Ambiguities by Zero or Near To Information) is designed to solve ambiguities related to insertion errors. As noted in Grudin [8] and Salthouse [20], the ambiguity lies in whether two keys were struck together or separately. The new method uses two rules, the Zero Time rule and the Impossible NT/CT-Mu rule to solve these ambiguities.

This method can be used for any text input methods that allow more than one key to be pressed simultaneously. Text input methods that only allow one character input at a time, such as stylus input, do not suffer from this particular ambiguity, and thus the method would not produce any additional information. The text input method should also allow the timing of key presses to be recorded, ideally to the nearest 0.001 seconds. Keyboards with a software key logger are ideal for use with this method, however, the method is still applicable to timings that were collected by other means such as video analysis.

The method also requires, as with most other categorisation methods, the presented text (PT) that was shown to the participant to copy, and the input stream (IS) of what the participant typed.

\subsection{Points of Insertion}

ReAZoN is based on the theory that, when an extra key press is inserted between two correct key presses, it can have three different 'points of insertion'. Below is a typical insertion error:

$$
\begin{aligned}
& \text { PT: floor } \\
& \text { IS: flootr }
\end{aligned}
$$

At the basic level of categorising errors into insertion, substitution and omission, there is no ambiguity as to whether the letter ' $t$ ' was inserted. However, more detailed categorisation is required if the cause of the error is to be found.

The inserted ' $t$ ' could have been inserted at three different "points of insertion":

1) ' $\mathrm{t}$ ' was typed at the same time as the second ' $\mathrm{o}$ ' in a single key press $(o->o t)$. 
2) ' $t$ ' was typed at the same time as the ' $r$ ' in a single key press $(r->t r)$.

3) ' $t$ ' was inserted on its own, between the ' $o$ ' and ' $r$ ' $((+) t)$.

Inspection of the Input Stream alone cannot tell us which of these points of insertion is correct. Without knowing this, we cannot say for sure whether this is an error of someone pressing two keys at the same time, or inserting a letter on its own. However, by analysing the timing at which the three letters ' $o$ ', ' $t$ ' and ' $r$ ' were pressed, it is possible to definitively determine which of the three possible points of insertion the error fits.

\subsection{Zero Time Rule}

By definition, a Zero Time keystroke refers to a keystroke where the time between it and the keystroke before it is shown as 0.000 seconds by the key logger. This can either be calculated by subtracting the two key presses time stamps, or can be automatically logged by software. In practice, the resolution of the logging software dictates the definition of Zero Time. If the resolution of the time logging is rounded to the nearest 0.001 seconds, this means that anything below 0.0005 seconds would be rounded down to 0.000 seconds, and anything equal to or above 0.0005 seconds will be rounded up to 0.001 seconds. This means that at this resolution, any two keys pressed with less than 1/2000 of a second in between will be classified as having a Zero Time gap between them. To exclude the time taken to read the presented text for the first time, the measuring of time starts once the first key is pressed for that phrase. Therefore, it is impossible for the first key press in a phrase to have zero time.

When two key presses have a key log of 0.000 seconds in between, then it is reasonable to assume that the two keys were pressed at exactly the same time. For example, consider the key $\log$ in Figure 1:

$$
\begin{aligned}
& {[00: 00.093] \mathrm{f}} \\
& {[00: 00.140] 1} \\
& {[00: 00.078] \mathrm{o}} \\
& {[00: 00.128] \mathrm{o}} \\
& {[00: 00.000] \mathrm{t}} \\
& {[00: 00.078] \mathrm{r}}
\end{aligned}
$$

Figure 1: Key Log of 'o' and 't' Pressed Simultaneously

The figure shows that ' $t$ ' was typed exactly at the same time as the 'o', as there was less than $1 / 2000$ of a second between the two keys being pressed down. It is possible to conclude from this that the point of insertion in this case was option 1) that the inserted letter was pressed at the same time as the letter in front of it $(o->o r)$

In contrast, Figure 2 shows a very similar key log, but the ' $r$ ' has an interval time of 0.000 seconds. This time, the point of insertion is case 2) the ' $t$ ' was typed at the same time as ' $r$ ' in a single key press $(r->t r)$.

$$
\begin{aligned}
& {[00: 00.093] \mathrm{f}} \\
& {[00: 00.140] 1} \\
& {[00: 00.078] \mathrm{o}} \\
& {[00: 00.128] \mathrm{o}} \\
& {[00: 00.084] \mathrm{t}} \\
& {[00: 00.000] \mathrm{r}}
\end{aligned}
$$

Figure 2: Key Log of 't' and ' $r$ ' Pressed Simultaneously Finally, consider Figure 3:
[00:00.093]f
$[00: 00.140] 1$
$[00: 00.078] \mathrm{o}$
[00:00.128]o
$[00: 00.084] \mathrm{t}$
[00:00.078]

Figure 3: Key Log of All Letters Pressed Individually

In this case, ' $t$ ' is considered to have been pressed on its own keystroke, separate from ' $\mathrm{o}$ ' or ' $\mathrm{t}$ ', as none of the letters involved have a zero time. It can be concluded that the points of insertion here was case 3 , that ' $t$ ' were inserted on its own $((+) t)$. In this case, it can be classified as Inserted Letter.

For options 1 and 2, although it is possible for two keys pressed down with a single keystroke to have a time interval larger than $1 / 2000$ of a second, any time interval greater than $1 / 2000$ of a second is considered to be a separate keystroke. It should also be noted that a keystroke having Zero Time does not clarify whether the typist used one finger to press down on two adjacent keys, or two fingers to press two keys at the same time.

\subsection{Impossible NT/CT Rule}

As already noted, Zero Time key presses can be used to demonstrate that the two keys were pressed at the same time, but cannot determine whether these were pressed by one finger or two. However, it is reasonable to assume that, if the two keys involved are adjacent to each other, the two keys were pressed by one finger that was misaimed, and landed in between the two keys.

An adjacent key to the intended key can be classified as either NT (Next-To) or CT (Close-To) according to its location relevant to the intended key (see Figure 4). NT keys are those keys that are directly next to the intended key, on the same row. CT keys are those keys adjacent to the intended keys but on a separate row. NT and CT keys are dependent on the keyboard layout (for example, QWERTY) and also on the particular model of the keyboard. Figure 4 shows, for a QWERTY keyboard, that if the intended key is ' $G$ ', then keys ' $\mathrm{F}$ ' and ' $\mathrm{H}$ ' are NT keys, and 'T', 'I', ' $\mathrm{V}$ ' and 'B' are $\mathrm{CT}$ keys.

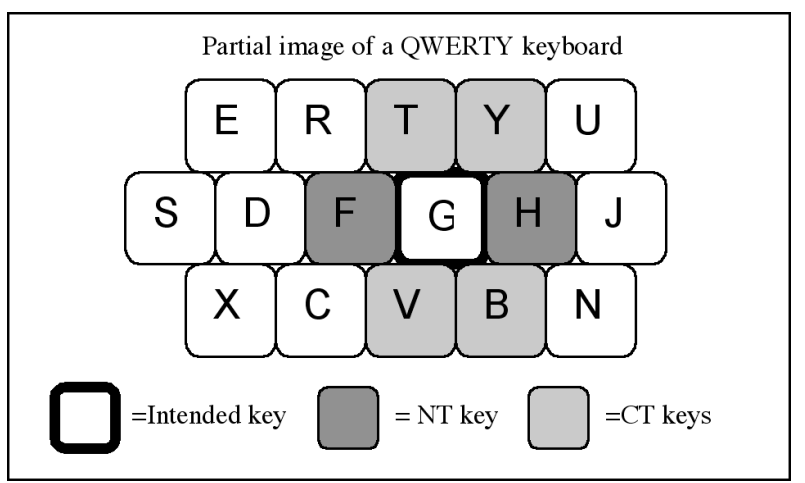

Figure 4: NT and CT Keys for 'G' on a QWERTY Keyboard

If the two keys had Zero Time interval, and they were NextTo or Close-To to each other, we can assume that the two keys were pressed with one finger and at the same time. Previously, in Figure 2, it was possible to say that ' $r$ ' and ' $t$ ' were pressed at the same time. As ' $r$ ' and ' $t$ ' keys are Next-To each other, it is now possible to conclude that these keys were pressed down simultaneously with one finger. 
It is also assumed that if the two keys involved are not adjacent to each other, it is impossible for one finger to press both keys in one keystroke. Therefore, a Zero Time key press, involving two non-adjacent keys will be assumed to have been pressed by two fingers. In Figure 1, ' $\mathrm{o}$ ' and ' $\mathrm{t}$ ' are neither Next-To nor Close-To each other, and so, although they were pressed at exactly the same time, two separate fingers made the two key presses.

By combining these two rules, it is possible to eliminate a large proportion of insertion ambiguities. It should be noted that the order in which the Zero Time analysis and the Impossible NT/CT-Mu analysis are carried out does not affect the number of ambiguities solved, so long as both analyses are carried out on all the data.

\section{THE STUDY}

Two large studies were conducted to gather phrase copy typing data for analysis from undergraduate students and young children. Phrase copying was preferred as it enables researchers to know what the participant intended to type ([15], [23]).

A four day study was carried out with undergraduate first years involving participants from the computer science department at the authors' university. A five day study was also carried out in three schools in Lancashire, England, with children from age 6 to 10 years old. Although there were some adjustments made between the students and the children in gathering personal information, the copy typing task itself was kept the same for the data to be comparable.

\subsection{Participants and Apparatus}

In the student study, out of the 92 participants, there were 80 male and 12 female students, majority (86) of whom were between the ages of $18-30$, with 4 others in their 30 s and 2 students in their 40s. All were studying courses in computing, with 18 studying at Foundation level and 74 were at Degree level. All experiments were carried out in quiet computing labs, equipped with the same model of keyboard (HP KB0316).

In the child study, of the 94 participants from 3 local schools, 58 were boys and 36 were girls. They were from year 2 (6 to 7 year olds), year 3 ( 7 to 8 year olds) and year 5 ( 9 to 10 year olds) with the majority of the participants ( 79 children) from year 5 .

\subsection{Design}

A new data-collecting tool called GetItAll was created to collect personal information about the participants, present the phrases and collect their typing data. It first asked for information about the participant, such as age, gender and whether they were left or right handed. The participants were then shown 10 phrases randomly selected from the 500 phrases found in the CPSet [11], which is suitable for use on both children and adults.

\subsection{Procedure}

In the student study, the investigator went into 7 practical classes of a computing module and asked for volunteers to take part. As with most computing departments, there were a higher proportion of male students (80) to female students (12). There were less than 20 students per lab, and each had access to a PC. The procedure was explained to them fully before they started the task.
The child study was part of a collection of studies carried out by a team of researchers. The children had an afternoon of various activities to do in rotation. The children had half an hour to carry out the typing in their schools' ICT suites in groups of 10 to 15 children, away from those carrying out other tasks. The procedure was explained to them fully beforehand and they worked on individual computers. As the child study was carried out in three schools, the equipment varied a little, with different makes and models of keyboards (Labtec Y-SAM64, Dell SK-8115 and HP KB-0136) However, they were all full sized, white letters on black keyboards with British keyboard layout.

The data collector was created using Visual Basic and can run on any Windows PC. As each participant ran individual copies of the program on their PC, and had 10 randomly selected phrases to copy. The phrases were shown one at a time in font style Verdana at size point 14, with a space for them to copy the phrase underneath (see Figure 5). The data collector logged what phrases were shown, each key that was pressed, its timestamp and time between the key presses.

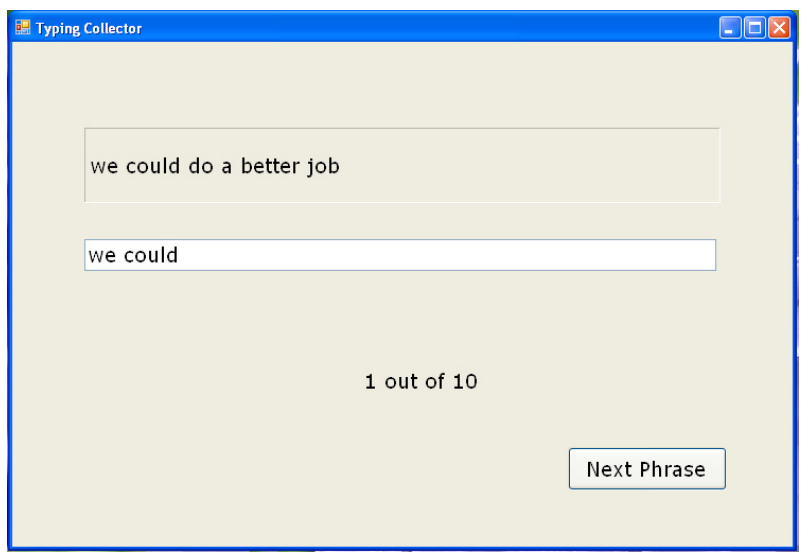

Figure 5: Screen Shot of the Data Collector Displaying a Phrase with the User Typing Directly Below

University of Central Lancashire, Faculty of Science and Technology Ethics Committee approved this study. Each participant gave verbal consent, and they were all made aware that they did not have to take part in the study. The participants were also told at the start, and reminded throughout the study, that they were free to stop participating if they wished to do so at any time. All participants chose to complete the tasks in full.

\subsection{Analysis Method}

\subsubsection{Initial Categorisation}

Firstly a manual analysis of the data was carried out to gauge the total number of errors made by participants. The errors were categorised based on the ExpECT method [12] of typing error categorisation.

The presented text (PT) was compared with the complete Input Stream (IS) as shown below:

$$
\begin{aligned}
& \text { PT: back to my seat } \\
& \text { IS: bach<k to m seaw }<\text { t }
\end{aligned}
$$

In this example, where a backspace is indicated by ' $<$ ', we see a variety of errors. The word 'back' was spelt with an ' $h$ ' then backspaced to fix it. A second error is 'my' typed without ' $y$ '. The word 'seat' is typed as 'seaw' and is quickly corrected. Therefore three errors were listed for this phrase.

Next, all ambiguous errors were listed, along with all the possible combination of keys pressed that could have 
produced the error. For example, the above error where 'back' was typed 'bach $<\mathrm{k}$ ' has the possibilities of:

1) ' $h$ ' was typed as ' $k$ ' (substitution)

2) ' $h$ ' was inserted (insertion)

3) 'c' was typed as 'ch' (insertion)

The second error in the example, where 'my' was typed as ' $m$ ', is not ambiguous, as the ' $y$ ' was clearly omitted. The third error ('seaw $<$ t' for 'seat') is ambiguous and therefore all possible combinations are again listed:

1) ' $w$ ' was inserted (insertion)

2) 'a' was typed as 'aw' (insertion)

3) ' $t$ ' was typed as ' $w$ ' (substitution)

Any ambiguous errors with a mixture of insertion, substitution and/or omission possibilities are considered to be a complex ambiguity. The two ambiguous errors used as examples here are complex ambiguities as they contain both insertion and substitution possibilities. If an ambiguous error consists of only insertion possibilities, it is an insertion-only ambiguity.

\subsubsection{The Impossible NT/CT-Mu}

The second stage of the analysis is to eliminate any ambiguities that contain impossible NT or CT combinations. In the first error ('bach' for 'back'), the third option for the error assumed that ' $c$ ' and ' $h$ ' were pressed with the same keystroke. However, 'c' and ' $h$ ' are neither Next-To nor Close-To each other, therefore, this possibility is ruled out from the list, leaving option 1 and 2 as the possible causes:
1) ' $h$ ' was typed has ' $k$ ' (substitution)
2) ' $h$ ' was inserted (insertion)
3) 'c' was typed as 'ch' (insertion)

In the error where 'seat' was typed as 'seaw', option 2 assumes that ' $a$ ' and ' $w$ ' was typed with the same keystroke. As these letters are Close-To each other, this option is not ruled out from the list. The possibility list for this error remains the same:
1) 'w' was inserted (insertion)
2) 'a' was typed as 'aw' (insertion)
3) 't' was typed as ' $w$ ' (substitution)

This elimination process was carried out on all the ambiguous errors listed in the first stage.

\subsubsection{Zero Time Analysis of Ambiguities}

The final stage of the analysis involved looking up the time logs for each of the insertion options to establish if the extra letter was individually inserted, or pressed along with another key. If the key press has Zero Time, it is assumed that it was pressed at the same time as the previous key press, although not necessarily with the same finger.

In the first example, it was already known that ' $c$ ' and ' $h$ ' were not pressed together by the same keystroke. However, the time log was checked, since it was possible that the two keys were pressed at the same time, with separate keystrokes. If the time between ' $c$ ' and ' $h$ ' was zero, then it is concluded that the two keys were pressed at the same time, with two separate fingers. This may indicate an error in the execution timing of these key presses, which could be investigated further at a later date. If the keys have greater than Zero Time in between, it is confirmed that the two keys were not pressed simultaneously. Although this does not reduce the number of possibilities, it does increase the reliability of the impossible NT/CT eliminations. The possibility list for the first error remains as:
1) ' $h$ ' was typed has ' $k$ ' (substitution)

2) ' $h$ ' was inserted (insertion)

3) 'e' was typed as 'ch' (insertion)

In the second example, the time between ' $a$ ' and ' $w$ ' was analysed. If this time is Zero Time, it was concluded that option 2, that 'a' was typed as 'aw' in the same keystroke was the cause of the error. Therefore, the possibility list for the second error was:

$$
\begin{aligned}
& \text { 1) 'w' was inserted (insertion) } \\
& \text { 2) 'a' was typed as 'aw' (insertion) } \\
& \text { 3) 't' was typed as 'w' (substitution) }
\end{aligned}
$$

This ambiguous error is now solved and listed as CT-Mu error.

On the other hand, if the time between was not Zero Time, it is concluded that the two keys were not pressed at the same time, and therefore option 2 was ruled out.

$$
\begin{aligned}
& \text { 1) ' } w \text { ' was inserted (insertion) } \\
& \text { z) ' } a \text { ' was typed as 'aw' (insertion) } \\
& \text { 3) ' } t \text { ' was typed as ' } w \text { ' (substitution) }
\end{aligned}
$$

This error is still considered ambiguous, but with less possibilities to consider.

Some ambiguities contain possibilities besides inserted letters:

$$
\begin{aligned}
& \text { PT: beach } \\
& \text { IS: eb<<beach }
\end{aligned}
$$

Here there are four possibilities, 1) 'b' and 'e' were swapped over (TE), 2) 'e' was inserted on its own, 3) space bar was pressed at the same time as 'e', 4) 'e' was pressed simultaneously with ' $b$ '. The ' $e$ ' key press has Zero Time, eliminating option 2 and 3 . The ' $b$ ' and ' $e$ ' keys are neither Next-To nor Close-To each other, eliminating option 4. Option 1 is the only remaining possibility, and thus the error is classified as Transposition Error (TE). This example shows that having Zero Time key press does not indicate that the two keys were pressed with a single keystroke, and that it is possible for two keystrokes to be made simultaneously. This is why it is important to look at the key combination to see if they fit under the Impossible NT/CT-Mu rule and Zero Time rule.

\subsection{Results}

Out of 622 errors found from the student study, 308 were ambiguous, and out of 951 errors found in the child study, 489 were ambiguous. Tables 3 and 4 summarise how many of these ambiguities were solved by the ReAZoN method. For both studies, the total number of ambiguities was counted. These were then classified into either an insertion-only ambiguity (an ambiguity with all the possibilities being insertions), or a complex ambiguity (an ambiguity with a combination of insertion, substitution and/or omission possibilities). The ReAZoN method was used to resolve or reduce ambiguities from both sets.

Table 3: Summary of Student Ambiguities Solved

\begin{tabular}{|l|l|l|l|l|}
\hline & & Solved & Reduced & Unchanged \\
\hline $\begin{array}{l}\text { Total number of } \\
\text { ambiguities }\end{array}$ & $\mathbf{3 0 8}$ & $\mathbf{1 0 4}$ & $\mathbf{1 5 3}$ & $\mathbf{5 1}$ \\
\hline $\begin{array}{l}\text { Insertion-only } \\
\text { ambiguities }\end{array}$ & 84 & 84 & 0 & 0 \\
\hline $\begin{array}{l}\text { Complex } \\
\text { ambiguities }\end{array}$ & 224 & 20 & 153 & 51 \\
\hline
\end{tabular}


Table 4: Summary of Child Ambiguities Solved

\begin{tabular}{|l|l|l|l|l|}
\hline & & Solved & Reduced & Unchanged \\
\hline $\begin{array}{l}\text { Total number of } \\
\text { ambiguities }\end{array}$ & $\mathbf{4 8 9}$ & $\mathbf{1 0 4}$ & $\mathbf{3 3 5}$ & $\mathbf{5 4}$ \\
\hline $\begin{array}{l}\text { Insertion-only } \\
\text { ambiguities }\end{array}$ & 84 & 84 & 0 & 0 \\
\hline $\begin{array}{l}\text { Complex } \\
\text { ambiguities }\end{array}$ & 405 & 16 & 335 & 54 \\
\hline
\end{tabular}

Student Data (Total Ambiguities $=308)$

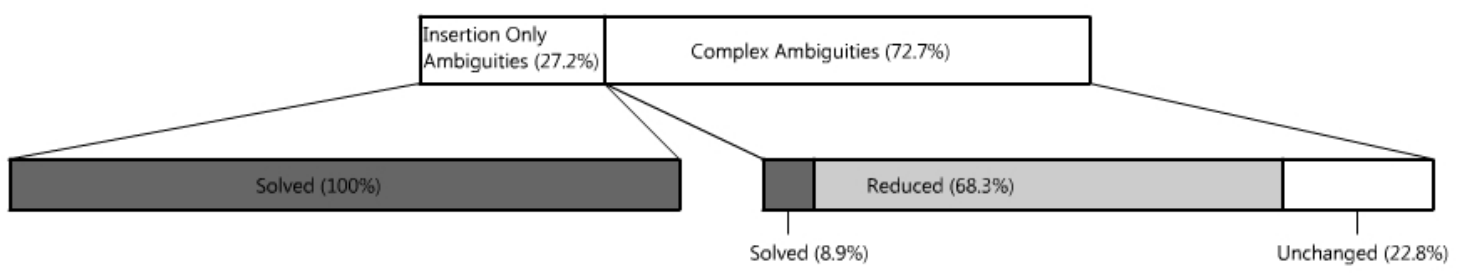

Child Data (Total Ambiguities $=489$ )

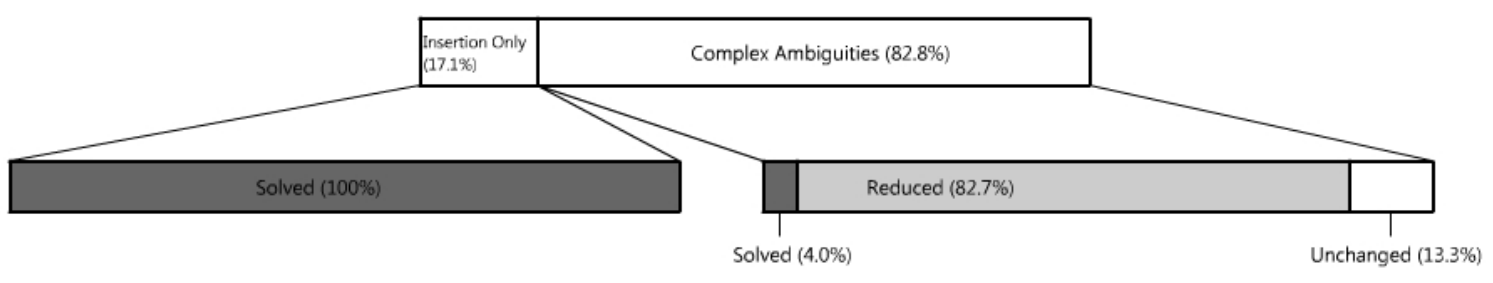

Figure 6: Percentage of Student and Child Ambiguities Solved by Zero Time Analysis and Impossible NT/CT-Mu Rule

\subsection{Discussion}

In both children and students, around half of the errors found were ambiguous. For the insertion-only ambiguities, the method was able to solve all of the ambiguities. Overall, the method solved a third of all student ambiguous errors and a fifth of child ambiguous errors. Although the child typing contained more ambiguous errors, less were solved in comparison to the student errors. This is due to the fact that children created less insertion-only ambiguous errors.

Besides the insertion-only ambiguities, the method was also able to solve small but significant number of complex ambiguities. These ambiguities often consisted of an insertion possibility, and either a substitution or omission possibility, so insertion possibility was eliminated.

Although the method did not solve the remaining complex ambiguities, it did eliminate at least one possibility each from $75-85 \%$ of the remaining complex ambiguous errors. Where the error contained more than one insertion possibility, the method was able to narrow them down to one insertion possibility. In some complex ambiguities, insertion possibilities were entirely eliminated.

In those ambiguities highlighted by Logan [14] (which were harder to solve as the errors were corrected immediately, and thus increasing the number of possibilities) the method was able to indicate whether two keys were inserted at the same time or inserted individually. Carrying out this method was simple, and gave consistent results, as it did not rely on
Figure 6 shows the percentage of ambiguous errors that were eliminated using the new method. Although the new method was able to resolve all the insertion-only ambiguities, in the case of complex ambiguities, only a small portion were resolved. However, a large portion (68-83\% of all complex ambiguities) had their possibilities reduced, which is highlighted in the lightest grey in Figure 6. 
whether the threshold for zero time analysis should remain at zero or be increased.

This new robust method can be incorporated into analysis of new keyboard designs such as on-screen keyboards and specialised keyboards, where large number of ambiguities have risen during analysis. The method will be particularly useful in solving ambiguities caused by small handheld devices where the ambiguous errors produced from the undersized keys are most suited for resolution by this method.

This method is a starting point of defining formal methods in solving all ambiguities, and future work is required in solving other types of ambiguities.

\section{REFERENCES}

[1] Berg, T. (2002), Slips of the Typewriter Key. Applied Psycholinguistics, 2002. 23: p. 185-207.

[2] Book, W. F. (1925), Learning to Typewrite. With a Discussion of the Psychology and Pedagogy of Skill. 1925: The Gregg Publishing Company.

[3] Cooper, W. E., ed. (1983), Cognitive Aspects of Skilled Typewriting. 1983, Springer-Verlag, New York.

[4] Dvorak, A., Merrick, N. L., Dealey, W. L., and Ford, G. C. (1936), Typewriting Behaviour. 1936: American Book Company, USA.

[5] Gentner, D. R. (1983), The Acqusition of Typewriting Skill. Acta Psychologica, 1983. 54: p. 233-248.

[6] Gentner, D. R., Grudin, J. T., Larochelle, S., Norman, D. A., and Rumelhart, D. E. (1983), A Glossary of Terms Including a Classification of Typing Errors, in Cognitive Aspects of Skilled Typewriting, W.E. Cooper, Editor. 1983, Springer Verlag. p. 39-43.

[7] Gentner, D. R., Grudin, J. T., Larochelle, S., Norman, D. A., and Rumelhart, D. E. (1984), A Glossary of Terms Including a Classification of Typing Errors, in Cognetive Aspects of Skilled Typewriting, W.E. Cooper, Editor. 1984, Springer Verlag. p. 39-43.

[8] Grudin, J. T. (1983), Error Patterns in Novice and Skilled Transcription Typing, in Cognitive Aspects of Skilled Typing, W.E. Cooper, Editor. 1983, SpringerVerlag: New York, USA. p. 121-139.

[9] Hayes, V., Wilson, G. D., and Schaffer, R. L. (1977), Typewriting Rate as a Function of Reaction Time. Perceptual and Motor Skills, 1977. 45: p. 1179-1184.

[10] Hiraga, Y., Ono, Y., and Hisao, Y. (1980), An Analysis of the Standard English Keyboard. in 8th Conference on Computational Linguistics. 1980. Tokyo, Japan.
[11] Kano, A., Read, J. C., and Dix, A. (2006), Children's Phrase Set for Text Input Method Evaluations, in Proceedings of the Fourth Nordic Conference on Human-Computer Interaction - NordiCHI. 2006, ACM Press: Oslo, Norway.

[12] Kano, A., Read, J. C., Dix, A., and MacKenzie, I. S. (2007), Expect: An Expanded Error Categorisation Method for Text Input, in HCI 2007. 2007, ACM: Lancaster, UK.

[13] Levenshtein, V. I. (1966), Binary Codes Capable of Correcting Deletions, Insertions and Reversals. Sovient Physics-Doklandy, 1966. 10: p. 707-710.

[14] Logan, F. A. (1999), Errors in Copy Typewriting. Journal of Experimental Psychology: Human Perception and Performance, 1999. 25(6): p. 17601773.

[15] MacKenzie, I. S. and Soukoreff, R. W. (2003), Phrase Sets for Evaluating Text Entry Techniques. in Extended Abstracts of the ACM Conference on Human Factors in Computing Systems - CHI 2003. 2003: ACM Press.

[16] MacKenzie, I. S. and Tanaka-Ishii, K., eds. (2007), Text Entry Systems: Mobility, Accessibility, Universality. 2007, Morgan Kaufmann Publishers: San Francisco.

[17] MacNeilage, P. F. (1964), Typing Errors as Clues to Serial Ordering Mechanisms in Language Behaviour. Language and Speech, 1964. 7: p. 1144-1159.

[18] Salthouse, T. A. (1984), Effects of Age and Skill in Typing. Journal of Experimental Psychology: General, 1984. 113(3): p. 345-371.

[19] Salthouse, T. A. (1985), Anticipatory Processing in Transcription Typing. Journal of Applied Psychology, 1985. 70(2): p. 264-271.

[20] Salthouse, T. A. (1986), Perceptual, Cognitive, and Motoric Aspects of Transcription Typing. Psychological Bulletin, 1986. 99(3): p. 303-319.

[21] Soukoreff, R. W. and MacKenzie, I. S. (2001), Measuring Errors in Text Entry Tasks: An Application of the Levenshtein String Distance Statistic. in Extended Abstract of the ACM Conference on Human Factors in Computing Systems (CHI). 2001. Seattle, WA: ACM: New York.

[22] White, W. T. (1945), Typing for Accuracy. 1945: H. M. Rowe Company, Balitimore \& Chicago, USA.

[23] Wobbrock, J. O. and Myers, B. A. (2006), Analyzing the Input Stream for Character- Level Errors in Unconstrained Text Entry Evaluations. in $A C M$ Transactions on Computer-Human Interaction (TOCHI). 2006: ACM. 